\title{
MDR1 C3435T AND C1236T POLYMORPHISMS IN CHILDHOOD ACUTE LYMPHOBLASTIC LEUKEMIA
}

Mona Wagdy Ayad, Niveen Lewis Mikhael, Nashwa Moustafa Saad Clinical and Chemical Pathology Department, Faculty of Medicine, Alexandria University, Egypt

Introduction:

Acute lymphoblastic leukemia (ALL) is the most common childhood malignancy worldwide.

Over the past four decades, the treatment of ALL among children has improved dramatically. Despite this success, drug resistance and treatment failure due to treatment-related toxicity still occur in about $20 \%$ of patients.

The multi-drug resistance 1 (MDR1) gene encodes a 170-kDa membrane transport protein called P-glycoprotein. One potential mechanism of drug resistance is mediated through the expression of the P-gp efflux pump, enabling ALL blasts to decrease intracellular toxic drug levels and thereby lower rates of apoptosis.

It was suggested that polymorphism of C3435T in exon 26 and C1236T in exon 12 of the MDR1 gene has a significant correlation with the P-gp expression level.

Aim of the work:

The aim of this work was to study MDR1 C3435T and MDR1 C1236T Polymorphisms in acute lymphoblastic leukemia patients and its relation to disease prognosis and response to treatment.

Patients:

Sixtychildrenadmitted to El ShatbyUniversity children's hospital and categorized into 2 groups; group I involved30patientswho were newly diagnosed with ALL, and group II involved 30apparently healthy age and sex matched subjects as a control group.

\section{Methods:}

All subjects were subjected to the following investigations: Thorough history taking, clinical examination, complete clinical and chemical investigations including $\mathrm{CBC}$, bone marrow examination, Immunophenotying for confirming diagnosis of pediatric acute lymphoblastic leukemia by flow cytometry, and Detection of MDR1 C3435T and MDR1 C1236T Polymorphisms in the studied patients \&controls by PCR-RFLP Assay.

\section{Results:}

Table (1) Genotype distribution of C3435T polymorphism of MDR1 gene

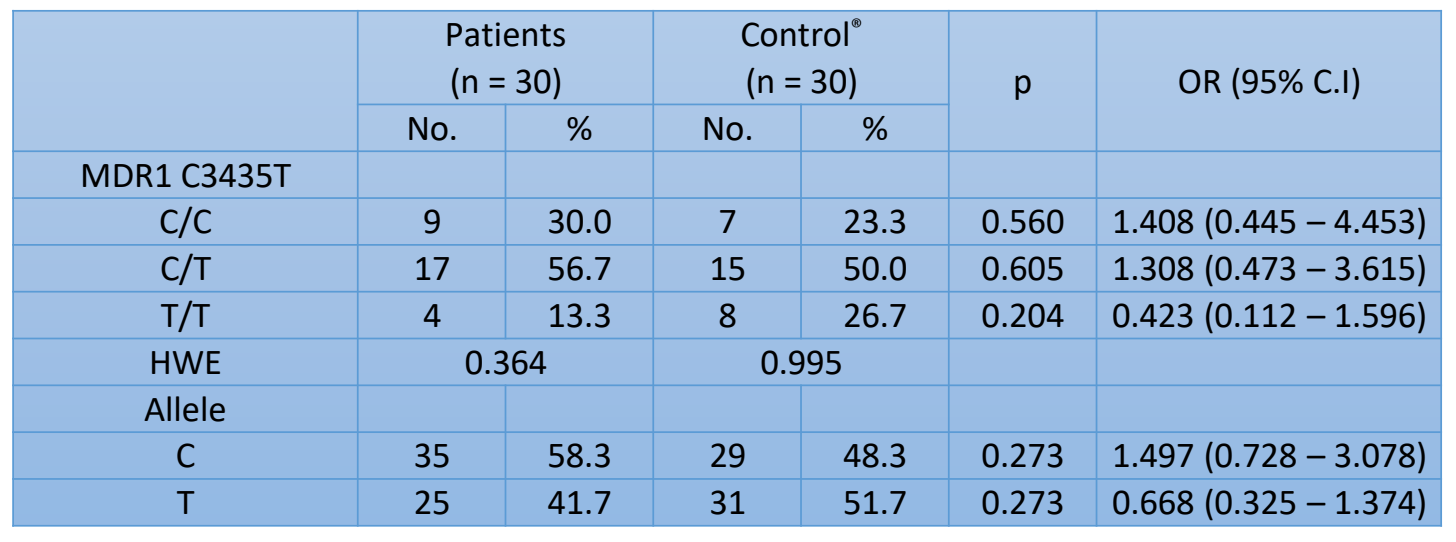

Table(2): Relation between MDR1 C3435T polymorphisms and outcomes in patients group after 1-year follow-up:

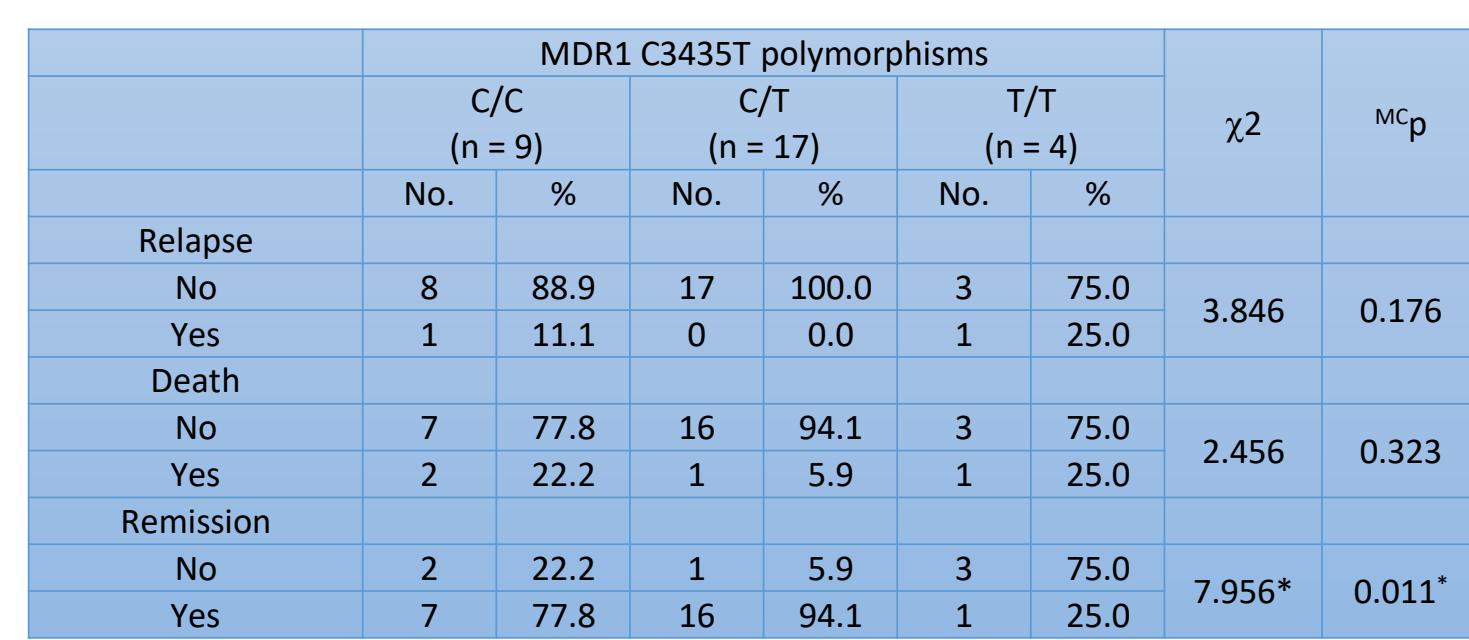

Table (3): Genotype distribution of C1236T polymorphism of MDR1 gene.

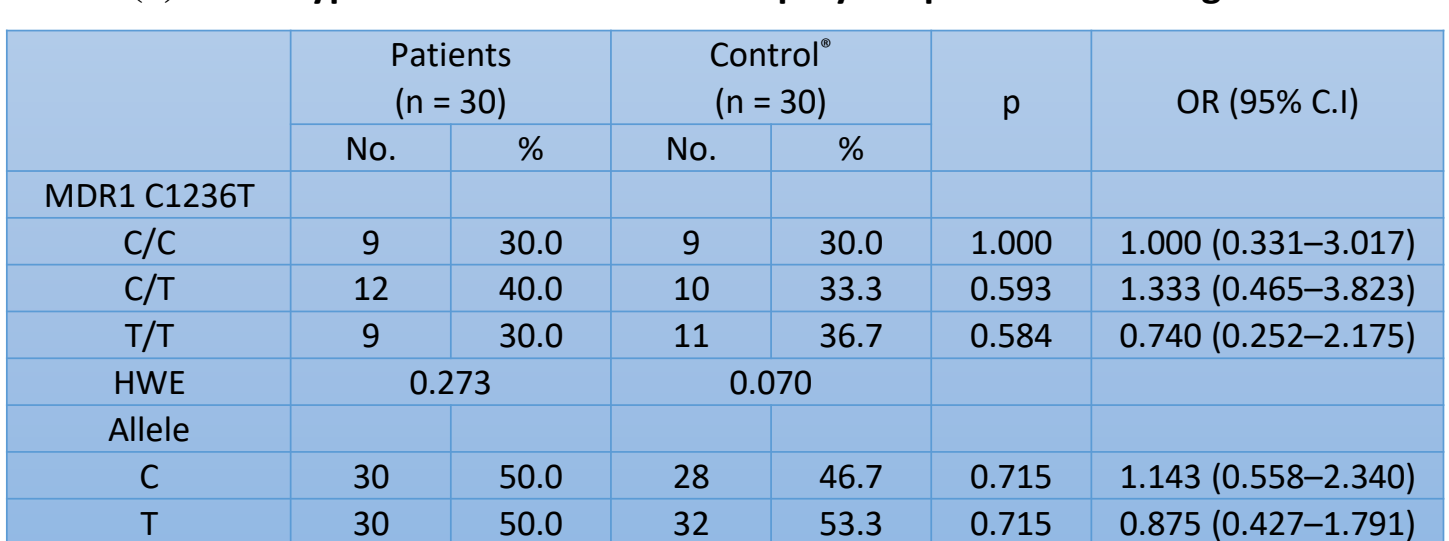

Table (4) Relation between MDR1 C1236T polymorphisms and outcomes in patients groupafter 1-year follow-up:

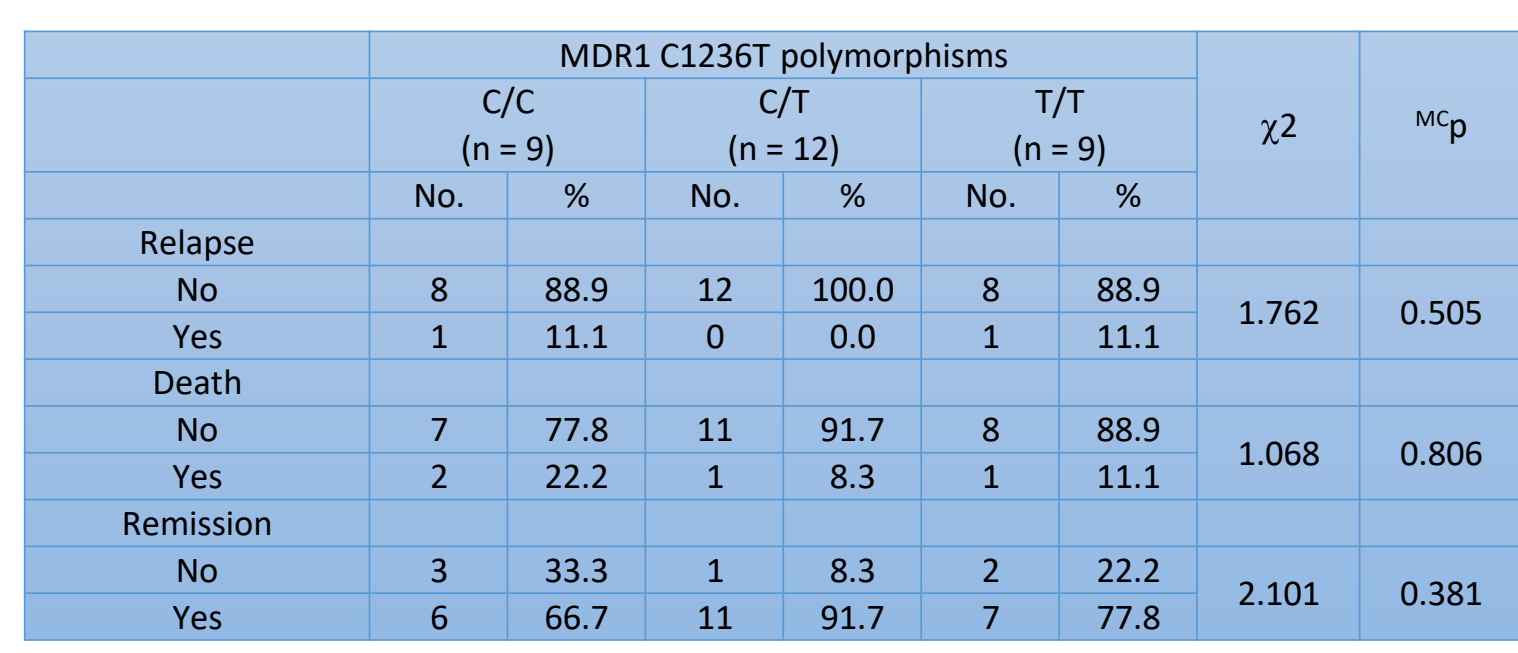

Conclusion:

From this study, we concluded that:

No distinct C3435T or C1236T genotype/allele increased the risk of developing childhood ALL.

T/T genotype of C3435T and C/C genotype of C1236T might be associated with a bad disease outcome and poor prognosis after treatment.

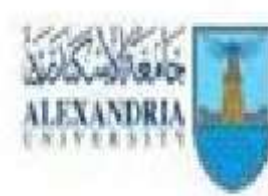
2021 Alexandria Faculty of Medicine 